\title{
Darf eine ASS-Therapie bei Ulkusblutungen fortgesetzt werden?
}

Eine ASS- Therapie ist nicht selten Ursache einer Schleimhautläsion im oberen Magen-Darm-Trakt mit konsekutiver Blutung. Ist diese mittels endoskopischer Therapie gestillt, stellt sich die Frage, ob die ASS-Therapie bis zur Abheilung der Läsion unterbrochen oder sofort weitergeführt werden sollte.

- Um diese Frage beurteilen zu können, wurden 156 Patienten mit einer Ulkusblutung randomisiert nach der endoskopischen Blutstillung entweder weiter mit Aspirin behandelt oder sie erhielten ein Placebo. Alle betroffenen Patienten erhielten während der ersten 72 Stunden Pantoprazol intravenös und anschließend oral. Endpunkte der Studie waren eine Ulkusrezidivblutung während der ersten 30 Tage, die Ge- samtmortalität und die Blutungsmortalität.

Während bei $10,3 \%$ der Patienten in der ASS-Gruppe eine Ulkusrezidivblutung auftrat, war dies in der Placebogruppe nur bei $5,4 \%$ der Fall. Allerdings hatten Patienten mit der nicht unterbrochenen ASS-Therapie eine geringere Gesamtmortalität im Vergleich zu den Placebopatienten (1,3\% vs. $12,9 \%)$. Insbesondere die kardiovaskuläre und zerebrovaskuläre Mortalität war in der Aspiringruppe signifikant niedriger.

\section{Kommentar}

Die Frage, ob bei einer oberen Gastrointestinalblutung die ASS-Therapie bei Patienten mit gesicherter Indikation nach entsprechender interventioneller Blutstillung sofort weitergeführt oder zunächst unterbrochen werden sollte, beschäftigt uns tagtäglich. Wie die Ergebnisse dieser Untersuchung zeigen, bewegen wir uns zwischen der Skylla der Rezidivblutung und der Charybdis eines erneuten kardio- oder zerebrovaskulären Ereignisses. Nach den Ergebnissen dieser Studie ist man eher geneigt, die ASS-Therapie nicht zu unterbrechen, um nicht ein solches gefährliches vaskuläres Ereignis zu provozieren. Für dieses Vorgehen spricht auch die Tatsache, dass im Falle einer Rezidivblutung diese erneut interventionell erfolgreich behandelt werden kann.

P. StiefelHAGEN

\section{- J.J. Y. Sung et al.}

Continuation of low-dose aspirin therapy in peptic ulcer bleeding: A randomised trial. Ann. Intern. Med. 152 (2010) 1-9

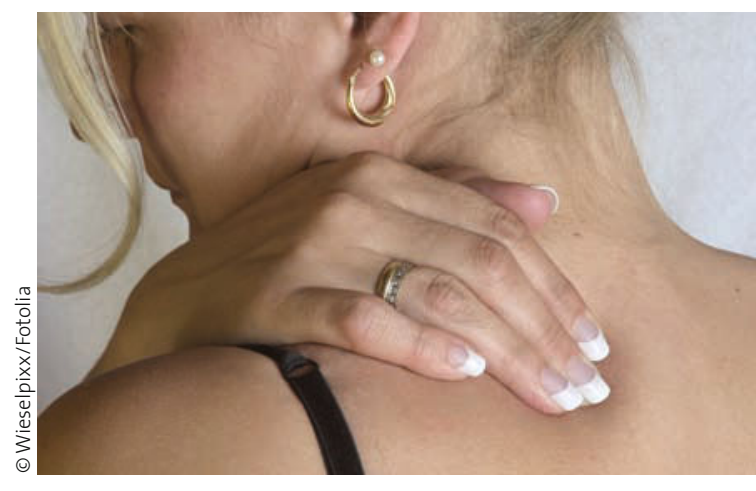

Kann ihr mit Akupunktur geholfen werden?

schmerzen nicht oder nur marginal effektiver ist als Placebobehandlung. Die Studie ist relativ gut gemacht. Zu bemängeln ist hauptsächlich die recht kleine Fallzahl. Das eingentlich Erstaunliche bei dieser Untersuchung ist jedoch die Schlussfolgerung der Autoren. Sie meinen, die Daten zeigten, dass die Wahl der gewählten Scheinakupunktur falsch war. Die fehlenden Gruppendifferenzen seien gemäß ihrer Meinung dadurch zu erklären, dass die Scheinbehandlung ebenso effektiv wie das Verum war. Die aus meiner Sicht viel offensichtlichere Konklusion, nämlich dass beide erhebliche Placeboeffekte induzieren, scheint ihnen nicht in den Sinn zu kommen - obschon es hierzu einen ganzen Berg von Daten gibt. Fazit: Akupunkturgläubige sind wohl nicht in der allerbesten Position, die Akupunktur wissenschaftlich zu testen. E. ERNST =

- N. Sahin et al.

Efficacy of acupunture in patients with chronic neck pain - a randomised, sham controlled trial. Acupunct. Electrother. Res. 35 (2010) 17-27 